\title{
Flow Properties of Moderately Concentrated Solutions of Polystyrene in Chlorinated Diphenyl
}

\author{
Kunihiro Osaki and Michio Kurata \\ Institute for Chemical Research, Kyoto University, Uji, Kyoto 611, Japan. \\ Mikio TAMura \\ Department of Industrial Chemistry, Kyoto University, Sakyo, Kyoto 606, Japan.
}

(Received January 13, 1970)

\begin{abstract}
Flow properties such as complex moduli, complex moduli with superimposed steady shear and non-Newtonian viscosity were measured on moderately concentrated solutions of polystyrene in chlorinated diphenyl. The range of molecular weight $M$ and of concentration $c$ was $5.6 \times 10^{5}-2.5 \times 10^{6}$ and $1-4.5 \mathrm{~g} / \mathrm{d} l$, respectively. The real part $G^{\prime}$ and the imaginary part $G^{\prime \prime}$ of the complex modulus of solutions with various molecular weights and concentrations were superimposed to give single composite curves, respectively, if $G^{\prime} M$ and $G^{\prime \prime} M$ were plotted against $\omega \eta M$, where $\omega$ is the angular frequency and $\eta$ is the zero-shear viscocity. Thus the steady shear compliance $J_{e}$ is proportional to $M$ and is independent of $c$. The values of the real part $G^{\prime}$ and the imaginary part $G^{\prime \prime}$ of the complex moduli with superimposed shear decreased when the rate of superimposed shear $\kappa$ increased. They decreased more rapidly in the range of low frequency than in the range of high frequency. The degree of decrease was larger for the real part than for the imaginary part. This effect of the superimposed steady shear on the complex moduli, together with the molecular weight and concentration dependence of the complex moduli, were qualitatively interpreted by assuming molecular aggregates in moderately concentrated polymer solutions.
\end{abstract}

KEY WORDS Polystyrene / Complex Modulus / Shear Flow / Molecular Weight/Concentration / Aggregate / Solution /

Recent studies have revealed that dynamic mechanical properties of polymers at infinite dilution in $\Theta$-solvent can be well interpreted by the theory based on a spring-beads model. ${ }^{1,2}$ The hydrodynamic interaction (h.d.i.) between polymer segments has been found extremely strong. On the other hand, the dynamic mechanical properties of dilute polymer solutions with finite concentrations seem to be described by the spring-beads model theories with varying degrees of hydrodynamic interaction. ${ }^{3-5}$ The hydrodynamic interaction seems to decrease as concentration, molecular weight or solvent power increases. However it seems unnatural to suppose that the nature of hydrodynamic interaction varies with concentration. This apparent variation may be due to interaction between polymer chains.

Frederick et al. employed the term "Rouse-like", (vanishing h. d. i.) or "Zimm-like" (infinite h. d.i.) behavior for the purpose of classifying different types of angular frequency dependence of the complex moduli. In this sense of terminology, the "Rouse-like" behavior represents the dependence illustrated by the heavy lines in Figure 1. These lines approach the relation $G^{\prime}=G^{\prime \prime}-\omega \eta_{S} \propto \omega^{1 / 2}$ at high frequencies where $G^{\prime}$ and $G^{\prime \prime}$ are the real and the imaginary parts of the complex modulus, respectively, $\omega$ is the angular frequency and $\eta_{\mathrm{s}}$ is the viscosity of the solvent. On the other hand, the "Zimm-like" behavior represents the dependence illustrated with thin lines in Figure 1, which approach the relation $1.7 G^{\prime}=G^{\prime \prime}-\omega \eta_{\mathrm{s}} \propto \omega^{2 / 3}$ at high frequencies. In this paper, we also use these terms "Rouse-like" and "Zimm-like" for the same purpose, regardless of the physical appropriateness of classifying the angular frequency de- 


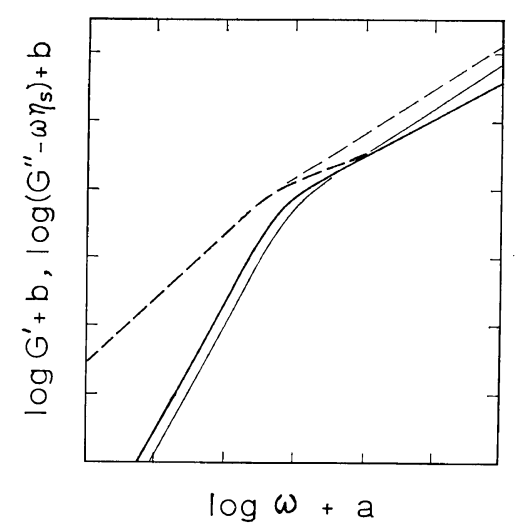

Figure 1. The real part $G^{\prime}$ and the imaginary part $G^{\prime \prime}-\omega \eta_{s}$ of the complex modulus plotted against the angular frequency $\omega$ in double logarithmic scale with arbitrary origin. Solid lines represent $G^{\prime}$ and broken lines $G^{\prime \prime}-\omega \eta_{s}$. Thick lines are for Rouse theory and thin lines for Zimm theory.

pendence of the complex modulus in terms of the hydrodynamic interaction.

In this paper, we will report the results of experiments designed to discover the origin of the "Rouse-like" behavior which becomes evident in dilute polymer solutions as concentration increases.

\section{EXPERIMENTAL}

Polystyrene samples were the same as used in a previous study. ${ }^{6}$ They were thermally polymerized at $115,80,60$, and $40^{\circ} \mathrm{C}$, respectively, and were designated as T 115, T 80, T 60 and T 40, respectively. Their molecular weights $M$ were $5.6 \times 10^{5}, 1.04 \times 10^{6}, 1.95 \times 10^{6}$, and $2.51 \times 10^{6}$, respectively as determined from the intrinsic viscosity in benzene. The solvent, Aroclor 1248 from Monsanto Chemical Company, was a mixture of chlorinated diphenyl with viscosity of 1.25 poise at $30^{\circ} \mathrm{C}$. The polymer solution was prepared by storing weighed amounts of the polymer and the solvent at $50^{\circ} \mathrm{C}$ and stirring occasionally with a spatula. The complex modulus, the complex modulus with superimposed steady shear and the steady shear viscosity were measured with a coaxial cylinder type rheometer. Details of measurement technique and apparatus were given in the previous paper. ${ }^{6}$ All experiments were performed at $30^{\circ} \mathrm{C}$.

\section{RESULTS AND DISCUSSIONS}

\section{Dynamic Mechanical Properties}

Experimental results for complex moduli are shown in Figures 2 and 3. In Figure 2, are given the log-log plots of the real part $G^{\prime}$ and the imaginary part $G^{\prime \prime}$ of complex moduli $v s$. the angular frequency $\omega$ for $3 \%$ solutions of polystyrene of various molecular weights. Obviously, the imaginary part $G^{\prime \prime}$ is almost proportional to $\omega$ for solutions of low molecular weight polystyrenes, T 115 and $\mathrm{T} 80$. For these solutions, no information is obtained which is useful for classifying the type of the dependence of the complex modulus on the angular frequency. However, for the solution of polystyrene T 60 both the real part $G^{\prime}$ and the imaginary part $G^{\prime \prime}$ of the complex modulus are proportional to $\omega^{1 / 2}$ and the relation $G^{\prime} \fallingdotseq G^{\prime \prime}$ holds good at high frequency so that this solution behaves "Rouse-like" in the sense stated in the

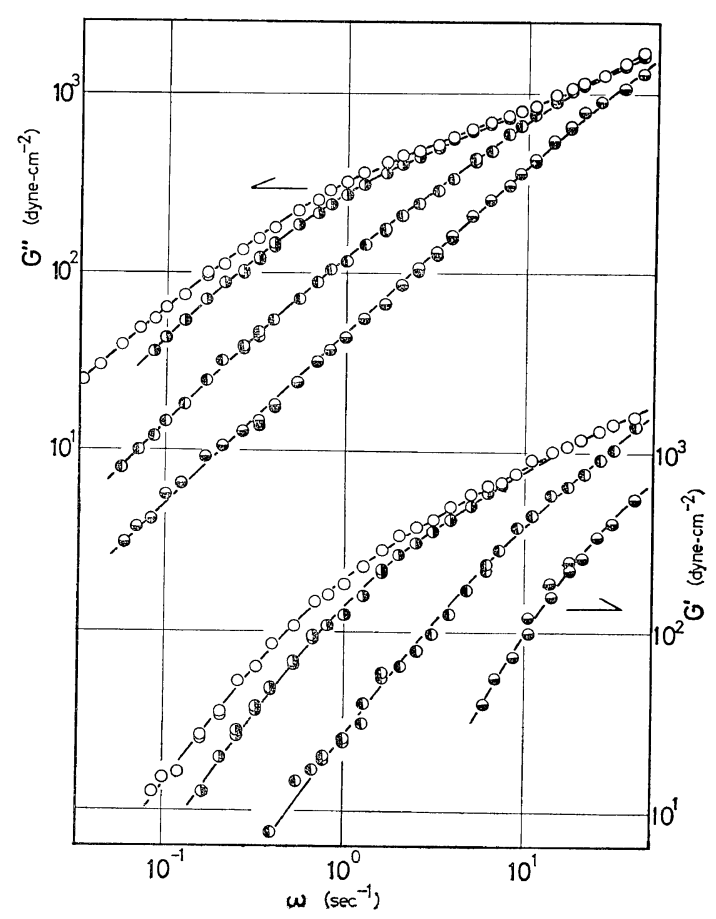

Figure 2. The real part $G^{\prime}$ and the imaginary part $G^{\prime \prime}$ of the complex modulus obtained for $3 \%$ solutions in Aroclor of polystyrene with various molecular weights. The molecular weights are $2.51 \times 10^{6}, 1.95 \times 10^{6}, 1.04 \times 10^{6}$ and $5.6 \times 10^{5}$, respectively, from top to bottom. 


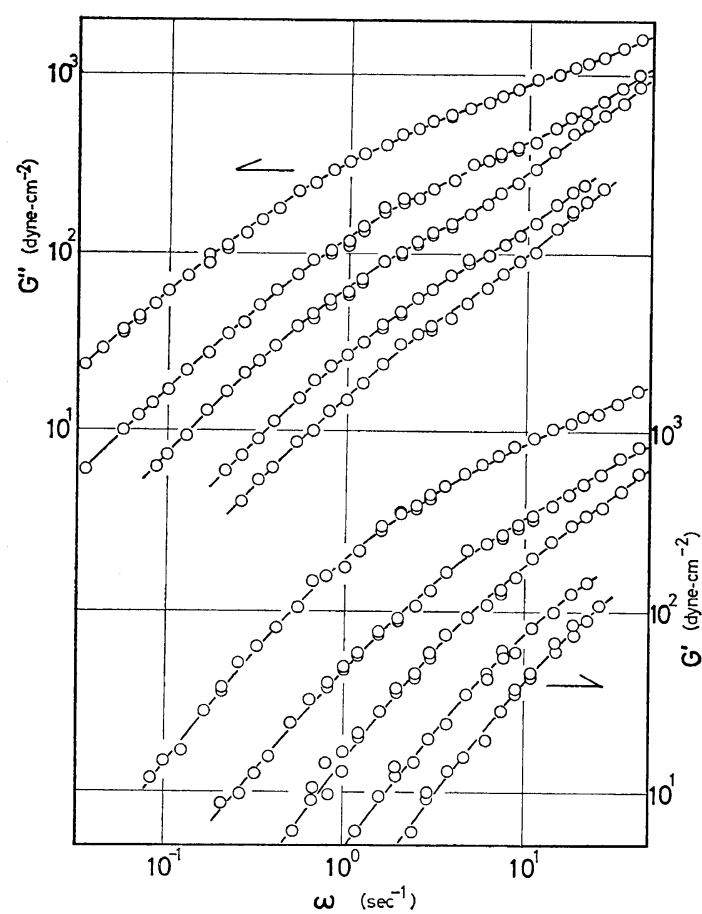

Figure 3. The real part $G^{\prime}$ and the imaginary part $G^{\prime \prime}$ of the complex modulus obtained for solutions of polystyrene $\mathbf{T} 40$ in Aroclor at various concentrations. The concentrations in $\mathrm{g} / \mathrm{d} l$ are $4.2,2.8$, 2.1, 1.4 and 1.05, respectively, from top to bottom.

introductory section. The solution of $\mathrm{T} 40$ is also very nearly "Rouse-like", except for two features: an inflexion point is observed in the plot of $\log G^{\prime \prime} v s$. $\log \omega$ at high frequency $(\omega=10)$ and $G^{\prime}>G^{\prime \prime}$ when $\omega$ is larger than $10 \mathrm{sec}^{-1}$. These two features are usually observed in concentrated solutions in which entanglement coupling is thought to exist. In Figure 3 are shown log-log plots of $G^{\prime}$ and $G^{\prime \prime} v s$. $\omega$ obtained for solutions of $\mathrm{T} 40$ with various concentrations. The angular frequency dependence of $G^{\prime}$ and $G^{\prime \prime}$ for the $2 \%$ solution is "Rouse-like". But $\omega$ is not high enough to classify the behavior of solutions of lower concentrations.

In order to see the effect of molecular weight $M$ and concentration $c$ more quantitatively, the data in Figures 2 and 3 are replotted in a reduced scale in which single composite curves for $G^{\prime}$ and $G^{\prime \prime}$ are obtained for various combinations of $M$ and $c$. That is to say, $G^{\prime}\left(M / M_{0}\right)^{\alpha}$ $\times\left(c_{0} / c\right)^{\beta}$ and $G^{\prime \prime}\left(M / M_{0}\right)^{\alpha}\left(c_{0} / c\right)^{\beta}$ are plotted against $\omega\left(\eta / \eta_{0}\right)\left(M / M_{0}\right)^{\alpha}\left(c_{0} / c\right)^{\beta}$ for various $M$ and $c$ to find two constants $\alpha$ and $\beta$ which give the best fit of the data for various combinations of $M$ and $c$. Here the quantities with the suffix zero mean the corresponding quantities for the standard system. We examined only integral and

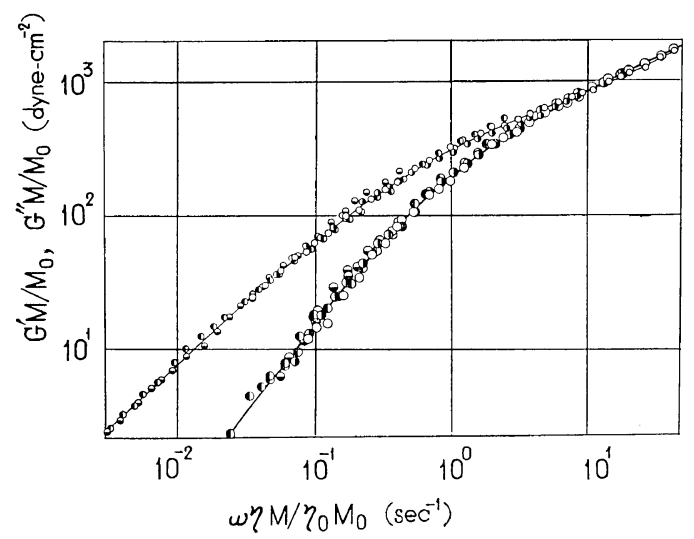

Figure 4. The real part $G^{\prime}\left(M / M_{0}\right)$ and the imaginary part $G^{\prime \prime}\left(M / M_{0}\right)$ of the reduced complex modulus plotted against the reduced angular frequency $\omega\left(M_{\eta} / M_{0} \eta_{0}\right)$ for the data shown in Figure 2 . The suffix zero indicates that the quantities are those for the sample $\mathbf{T} 40$. Different types of circles represent different molecular weights of the polymer: $\bigcirc, 2.51 \times 10^{6} ; 1.95 \times 10^{6} ; 0,1.04 \times 10^{6}$, and $5.6 \times 10^{5}$.

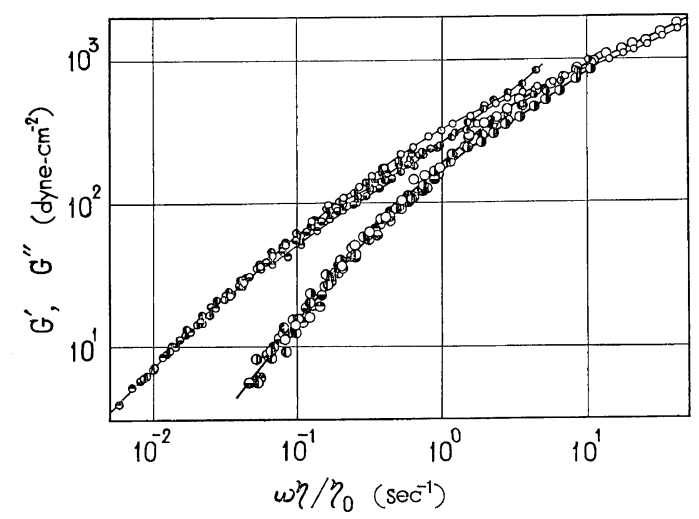

Figure 5. The real part $G^{\prime}$ and the imaginary part $G^{\prime \prime}$ of the complex modulus plotted against the reduced angular frequency $\omega \eta / \eta_{0}$ for the data shown in Figure 3, where $\eta_{0}$ represents the viscosity of $3 \%$ solution. Different types of circles represent different concentrations: $\bigcirc, 4.2 \mathrm{~g} / \mathrm{d} l ;(2.8 \mathrm{~g} / \mathrm{d} l$; $\bigcirc, 2.1 \mathrm{~g} / \mathrm{d} l ; \infty, 1.4 \mathrm{~g} / \mathrm{d} l$ and $\odot, 1.05 \mathrm{~g} / \mathrm{d} l$. 
half integral numbers for $\alpha$ and $\beta$ because the data are not precise enough to allow for their more precise determination. Then it is not difficult to find the best values of $\alpha$ and $\beta$ to be $\alpha=1$ and $\beta=0$ for the data given in Figures 2 and 3. The reduced plot of the data in Figure 2 with $\alpha=1$ is shown in Figure 4 and that of the data in Figure 3 with $\beta=0$ in Figure 5, in which the $3 \%$ solution of T 40 is selected as the standard system. It is obvious from Figure 4 that the data for solutions with various molecular weights and with the same concentration can be superimposed on a single composite curve with $\alpha=1$. This result means that the shape of the relaxation spectrum is the same for various molecular weights if the concentration be kept constant. The behavior of the $3 \%$ solutions may well be classified as "Rouse-like". For solutions of $\mathrm{T} 40$ of various concentrations, the reduction is not so good. This probably indicates that the shape of the relaxation spectrum varies with varying concentration.

It may be easily seen that the dependence of the steady shear compliance on $M$ and $c$ is $M^{\alpha} / c^{\beta}$ if the reduction is successfully performed at low frequency. Thus for moderately concentrated solutions of polystyrene in chlorinated diphenyl,

$$
J_{\mathrm{e}} \propto M^{1} / c^{0} .
$$

As is well known, the steady shear compliance $J_{e}$ increases with increasing concentration in the range of very low concentration, takes a maximum value and decreases in the range of high concentration. ${ }^{7}$ The relation 1 shows that the range of concentration of the present study corresponds to the maximum of $J_{\mathrm{e}}$ and lies between "dilute" and "concentrated" regions.

Dynamic moduli with superimposed steady shear

In Figures 6 and 7 are shown the real part $G_{\kappa}{ }^{\prime}$ and the imaginary part $G_{\kappa}{ }^{\prime \prime}$ of the complex modulus with superimposed steady shear obtained for $2 \%$ and $3 \%$ solutions, respectively, of polystyrene T40 in chlorinated diphenyl. For the sake of clarity of figures, the experimental points are shown only for the case of $2 \%$ solution with the superimposed shear rate $\kappa=1.2 \mathrm{sec}^{-1}$. For the remaining cases, smoothed curves of $\log G_{k}{ }^{\prime}$ and $\log G_{\kappa}{ }^{\prime \prime}$ are indicated as functions of $\log \omega$. The effect of the superimposed steady shear is

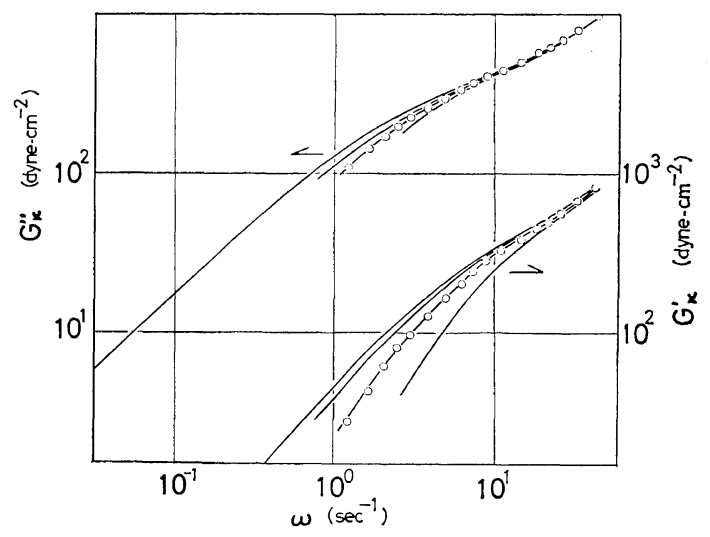

Figure 6. The real part $G_{x^{\prime}}$ and the imaginary part $G_{\kappa}{ }^{\prime \prime}$ of the complex modulus with superimposed steady shear obtained for $2 \%$ solution of polystyrene T 40 in Aroclor. The rate $\kappa$ of superimposed steady shear in $\sec ^{-1}$ is $0,0.49,1.2$, and 3.8 , respectively, from left to right. Measured values are shown with circles only for $\kappa=1.2$.

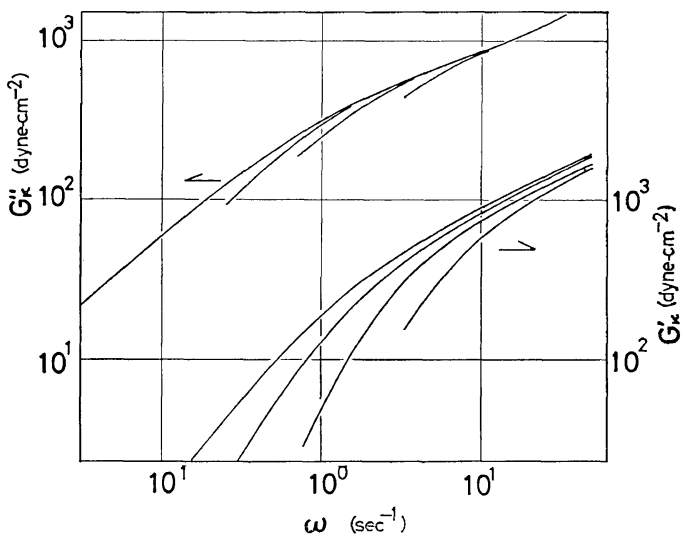

Figure 7. The real part $G_{\kappa}{ }^{\prime}$ and the imaginary part $G_{\kappa}{ }^{\prime \prime}$ of the complex modulus with superimposed steady shear obtained for $3 \%$ solution of polystyrene T 40 in Aroclor. The rate $\kappa$ of superimposed steady shear in $\sec ^{-1}$ is $0,0.51,1.5$, and 4.5 , respectively, from left to right.

to diminish magnitudes of both the real part and the imaginary part of the complex modulus in the whole range of $\omega$. The degree of the effect of the superimposed shear is different for the real part and for the imaginary part and for different ranges of the angular frequency as follows:

(i) The effect of the superimposed shear is 
larger for the real part of the complex modulus than for the imaginary part.

(ii) The effect of the superimposed shear is larger in the range of lower angular frequency.
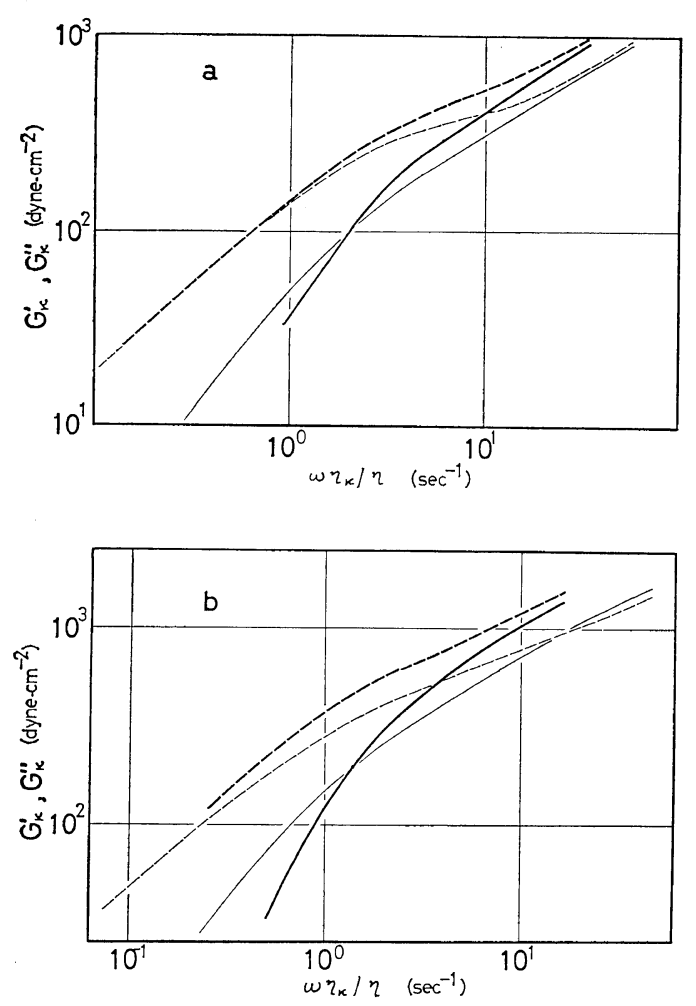

Figure 8. The real part $G_{\kappa}{ }^{\prime}$ and the imaginary part $G_{k}{ }^{\prime \prime}$ of the complex modulus with superimposed steady shear plotted against the reduced angular frequency $\omega \eta_{\kappa} / \eta$. Solid lines represent $G_{\kappa}{ }^{\prime}$ and the broken lines $G_{\kappa}{ }^{\prime \prime}$. Thin lines correspond to $\kappa=0$ and thick lines to the finite value of the rate of superimposed steady shear. (a) The result for $2 \%$ solution of polystyrene T 40 and $\kappa=1.5$ $\mathrm{sec}^{-1}$ for thick lines, (b) the result for $3 \%$ solution of polystyrene $\mathrm{T} 40$ and $\kappa=1.2 \mathrm{sec}^{-1}$ for thick lines.

Thus in the range of the highest frequency the imaginary part is practically independent of the superimposed shear. The inflection point observed on the curve of $\log G^{\prime \prime}$ vs. $\log \omega$ in Figure 7 becomes indistinct and disappears as the rate of the superimposed steady shear is increased. Moreover, in Figure $7, G^{\prime}$ is larger than $G^{\prime \prime}$ at high frequency. This is to be compared with the fact that $G_{\kappa}{ }^{\prime}$ is smaller than $G_{\kappa}{ }^{\prime \prime}$ even at high frequency if $\kappa$ is sufficiently high (see also Figure 8 ). Thus the trace in $3 \%$ solution of $\mathrm{T} 40$ of the feature of concentrated polymer solutions disappears with the superposition of the steady shear. At first sight the effect of the increase in the rate of shear is similar to the effect of decrease in the molecular weight but precise observation reveals that this is not the case.

In order to see the effect of the superimposed shear on the shape of the curves, some of the data in Figures 6 and 7 are replotted in Figures $8 \mathrm{a}$ and $8 \mathrm{~b}$, respectively: $\log G_{\kappa}{ }^{\prime}$ and $\log G_{\kappa}{ }^{\prime \prime}$ are plotted against $\log \left(\omega \eta_{\kappa} / \eta\right)$, selected values of $\kappa$ being 0 and $1.5 \mathrm{sec}^{-1}$ in Figure $8 \mathrm{a}$ and 0 and 1.2 in Figure $8 b$. Here $\eta$ is the zero-shear viscosity and $\eta_{k}$ is the steady shear viscosity at the rate $\kappa$ of the superimposed shear. It may be seen that the curves for $\kappa=1.5$ in Figure $8 \mathrm{a}$ or for $\kappa=1.2$ in Figure $8 \mathrm{~b}$ cannot be superposed on the curves for $\kappa=0$ by any parallel shift. Thus the shape of the curves changes with increasing rate of the superimposed shear. Apparently the superimposed shear changes "Rouselike" curves into "Zimm-like" curves. It is also to be noted that this change takes place at such a low rate of shear as $\kappa=1 \mathrm{sec}^{-1}$.

Steady Shear Viscosity

Steady shear viscosities $\eta_{k}$ at various rates of shear $\kappa$ are plotted against the molecular weight $M$ in Figure 9 and against the concentration $c$ $\mathrm{g} / \mathrm{d} l$ in Figure 10 . The zero-shear viscosity is proportional to about 1.8 -th power of $M$. At a

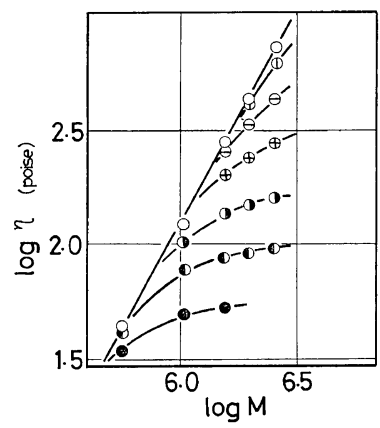

Figure 9. The viscosity at fixed rate of shear $\eta_{k}$ for $3 \%$ solution of polystyrene in Aroclor plotted against the molecular weight of the polymer. The rate of shear $\kappa\left(\mathrm{sec}^{-1}\right)$ is $0,0.3,1,3,10,30$, and 100 , respectively, from top to bottom. 


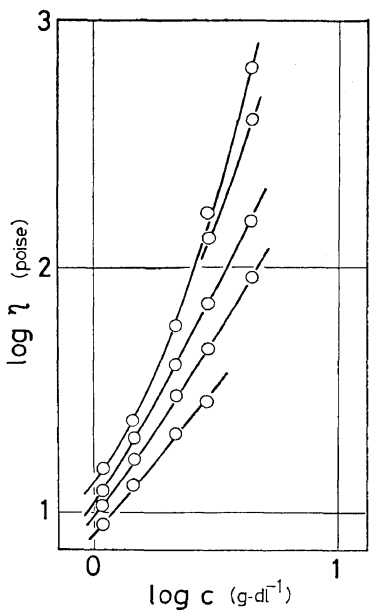

Figure 10. The viscosity at fixed rate of shear $\eta_{k}$ for solutions of polystyrene T 40 in Aroclor plotted against the concentration. The rate of shear $\kappa\left(\mathrm{sec}^{-1}\right)$ is $0,1,10,30$, and 100 , respectively, from top to bottom.

fixed value of $\kappa$, the viscosity $\eta_{\kappa}$ remains pracitcally unchanged from $\eta$ until the molecular weight reaches a certain value. The non-Newtonian effect appears abruptly above this value of molecular weight. In the non-Newtonian region, it is remarkable that the viscosity $\eta_{k}$ is proportional to a very low power of $M, e . g ., 0.5$-th power at $M, 2 \times 10^{6}$ and $\kappa, 1 \mathrm{sec}^{-1}$, and becomes even independent of $M$ at sufficiently high values of $M$ and $\kappa$. Such a tendency has been found in many concentrated and bulk polymer system, ${ }^{8-11}$ but in the range of higher values of $\kappa$. As for the dependence on concentration, the zero-shear viscosity is not proportional to a constant power of $c$ but varies as $c^{2}-c^{5}$ dependent on the range of concentration. As the rate of shear is increased, the curve of $\log \eta_{k} v s . \log c$ approaches a straight line, and the slope of the line approaches unity. Thus the viscosity at a fixed rate of shear seems to satisfy the relation $\eta_{\kappa} \propto M^{0-0.5} c$ when $\kappa$ is high and $M$ is not very low.

\section{Some speculations on the results}

The variation of the shape of curves with increasing concentration in Figure 3 may be explained by assuming that the longest time end of the distribution function of relaxation times becomes of box-type in high concentration regions. Appearance of the inflection point in $G^{\prime \prime}$ supports this conjecture. ${ }^{12}$ With this assumption the variation of the shape of curves with increasing rate of shear in Figure 6 or 7 suggests the disappearance of the box-type spectrum with increasing rate of shear. The remarkable decrease in viscosity with increasing rate of shear in Figure 9 is also attributed to disappearance of the boxtype spectrum. This interpretation is on the same line as that previously given for concentrated polystyrene solutions. ${ }^{6}$ However, it may be interesting here to give another line of interpretation.

Theories in dilute polymer solutions predict that $J_{\mathrm{e}}\left[\eta /\left(\eta-\eta_{\mathrm{s}}\right)\right]^{2}$ is inversely proportional to the number of polymer molecules in unit volume of the solution, that is

$$
J_{\mathrm{e}}\left(\frac{\eta}{\eta-\eta_{\mathrm{s}}}\right)^{2} \propto M / c
$$

In the solutions treated here, we can always put $\eta \gg \eta_{\mathrm{s}}$ and so rewrite Eq. 1 in the form that

with

$$
J_{\mathrm{e}}\left(\frac{\eta}{\eta-\eta_{\mathrm{s}}}\right)^{2} \propto M^{*} / c
$$

$$
M^{*} \propto c M
$$

This is equal to assuming that in moderately concentrated polymer solutions each molecule is elastically coupled with other molecules through the so-called "entanglements" and it behaves like an aggregated quasi-particle in flow whose "molecular weight" is $M^{*}$. Then, it may be natural to assume that the effective molecular weight $M^{*}$ is proportional to both the real molecular weight $M$ and the concentration of partners $c$. In this interpretation, the shape of the reduced curves of $G^{\prime}$ and $G^{\prime \prime}$ shown in Figures 4 and 5 suggests that the aggregates are nearly "Rouse-like" particles in contrast with original molecules which are generally "Zimmlike" particles. As the steady shear is superimposed and its rate is increased, the aggregates may decompose towards the original molecules as a result of breaking of entanglements. The dynamic mechanical property thus changes from the "Rouse-like" to "Zimm-like" behavior with increasing rate of shear as observed. This interpretation is of course only a conjecture unless its theoretical basis is given. 
Acknowledgement. The authors are obliged to Mr. Kazumasa Matusita who performed some of the dynamic measurements.

\section{REFERENCES}

1. H. Tanaka, et al., Rept. Progr. Polym. Phys., Japan, 7, 145 (1964); A. Sakanishi, J. Chem. Phys., 48, 3850 (1968).

2. B. H. Zimm, J. Chem. Phys., 24, 266 (1956).

3. J. E. Frederick, et al., J. Phys. Chem., 68, 1974 (1964).

4. N. W. Tschoegl, J. Chem. Phys., 39, 149 (1963); 40, 473 (1964).

5. P. E. Rouse, J. Chem. Phys., 21, 1272 (1953).
6. K. Osaki, et al., J. Phys. Chem., 69, 4183 (1965).

7. See for example, L. A. Holmes, and J. D. Ferry. J. Polym. Sci., Part C, 23, 291 (1968).

8. R. S. Porter and J. H. Johnson, Trans. Soc. Rheol. 6, 107 (1962).

9. H. P. Schreiber, et al., Polymer, 4, 355, 365 (1963).

10. S. N. Chinai and W. C. Schneider, J. Polym. Sci., Part A, 3, 1359 (1965).

11. T. Kataoka and S. Ueda, J. Polym. Sci., Part $A$, 3, 2947 (1965).

12. M. Yamamoto and H. Tanaka, J. Soc. Mat. Sci. Japan (Zairyo), 15, 307 (1966); J. Macromol. Sci. (Phys.), B1, 199 (1967). 